\title{
The Outflow Ranking Method for Weighted Directed Graphs
}

René van den Brink ${ }^{7}$

Robert P. Gilles ${ }^{2}$

' Department of Econometrics, Vrije Universiteit Amsterdam, and Tinbergen Institute.

2 Department of Economics, Virginia Tech, Virginia, USA, and visitor Tinbergen Institute. 


\section{Tinbergen Institute}

The Tinbergen Institute is the institute for economic research of the Erasmus Universiteit Rotterdam, Universiteit van Amsterdam, and Vrije Universiteit Amsterdam.

Tinbergen Institute Amsterdam

Roetersstraat 31

1018 WB Amsterdam

The Netherlands

Tel.: $\quad+31(0) 205513500$

Fax: $\quad+31(0) 205513555$

Tinbergen Institute Rotterdam

Burg. Oudlaan 50

3062 PA Rotterdam

The Netherlands

Tel.: $\quad+31(0) 104088900$

Fax: $\quad+31(0) 104089031$

Please send questions and/or remarks of nonscientific nature to driessen@tinbergen.nl.

Most TI discussion papers can be downloaded at http://www.tinbergen.nl. 


\title{
The Outflow Ranking Method for Weighted Directed Graphs*
}

\author{
René van den Brink ${ }^{\dagger} \quad$ Robert P. Gilles ${ }^{\ddagger}$
}

May 2006

\begin{abstract}
A ranking method assigns to every weighted directed graph a (weak) ordering of the nodes. In this paper we axiomatize the ranking method that ranks the nodes according to their outflow using four independent axioms. This outflow ranking method generalizes the ranking by outdegree for directed graphs. Furthermore, we compare our axioms with other axioms discussed in the literature.
\end{abstract}

Keywords: Decision analysis, Weighted directed graph, Ranking method.

JEL subject classification: C65, D71.

AMS subject classification: 05C20, 91B14.

*Financial support for this research was provided by the Netherlands Organization for Scientific Research, grant \#B45-282.

${ }^{\dagger}$ Corresponding author: Department of Econometrics and Tinbergen Institute, Free University, Amsterdam, the Netherlands. Email: jrbrink@feweb.vu.nl.

${ }_{\ddagger}^{\ddagger}$ Department of Economics, Virginia Tech, Blacksburg, Virginia, USA. Email: rgilles@vt.edu. 


\section{Introduction}

A weighted directed graph—or weighted digraph—is a pair $(N, \omega)$ where $N=\{1, \ldots, n\}$ is a finite set of $n$ nodes and $\omega: N \times N \rightarrow \mathbb{R}_{+}$is a weight function assigning nonnegative weights to every ordered pair of nodes in $\mathrm{N}$. A dominance structure between agents or alternatives such that the pairwise dominance relations differ in 'strength' can be represented by such weighted digraphs. Here, the nodes in N represent these agents or alternatives and the value $\omega(i, j)$ is a measure of how strongly $i \in N$ dominates $j \in N$. We only consider weighted digraphs $(N, \omega)$ that satisfy $\omega(i, i)=0$ for every $i \in N$. Since we assume the set of nodes $N$ to be given, we may represent each weighted digraph $(N, \omega)$ by its weight function $\omega$. The family of all weighted digraphs on $\mathrm{N}$ is indicated by $\mathcal{W}$. (Note that for any $\omega \in \mathcal{W}$ it is allowed that $\omega(i, j) \cdot \omega(j, i) \neq 0$ as long as $i \neq j$, i.e. it might be that two distinct nodes dominate each other.) The outflow of node $i$ in weighted digraph $\omega$ is given by $\sigma_{i}^{\text {out }}(\omega)=\sum_{j \in N} \omega(i, j)$.

A preorder on $N$ is a relation $\mathfrak{R} \subseteq N \times N$ that is reflexive (i.e., $(i, i) \in \mathfrak{R}$ for all $i \in N$ ) and transitive (i.e., $\{(i, j),(j, h)\} \subseteq \Re$ implies $(i, h) \in \Re$ for every triple $i, j, h \in N$ ). (See, e.g., Bourbaki [1], page 133.) A preorder $\mathfrak{R}$ on $N$ is complete if $\{(i, j),(j, i)\} \cap \Re \neq \emptyset$ for every pair $i, j \in N, i \neq j$. Throughout this paper we limit our discussion to complete preorders. Using standard notation for a complete preorder $\Re$ we denote $i \succcurlyeq j$ if and only if $(i, j) \in \Re$. Further we denote $i \succ j$ if and only if $i \succcurlyeq j$ and not $j \succcurlyeq i$, and we denote $i \sim j$ if and only if $i \succcurlyeq j$ as well as $j \succcurlyeq i$. If $i \succcurlyeq j$ then we say that node $i$ is "ranked at least as high" as node $j$, while if $i \succ j$ we say that $i$ is "ranked higher" than $j$. If $i \sim j$ then $i$ and $j$ are "ranked equally". We denote the family of all complete preorders on the set $N$ by $\mathcal{P}$.

A ranking method is a mapping $\mathrm{R}: \mathcal{W} \rightarrow \mathcal{P}$ which assigns to every weighted digraph $\omega \in \mathcal{W}$ a complete preorder $R(\omega) \in \mathcal{P}$. With slight abuse of notation we use the convention that a ranking method is also represented by $\left\{\succcurlyeq_{\omega} \mid \omega \in \mathcal{W}\right\} \subseteq \mathcal{P}$ where $i \succcurlyeq_{\omega} j$ if and only if $(i, j) \in R(\omega)$.

Various ranking methods for weighted digraphs can be found in the literature. For example, the method of ranking by net flow (see Bouyssou [2]) ranks the nodes according to the difference between their total outflow and total inflow. Bouyssou and Perny [3] characterize a partial ranking method (i.e., a method that assigns to every weighted digraph a preorder that is not necessarily complete) in which node $i$ is ranked at least as high as another node $j$ if and only if the outflow of $i$ is at least as high as the outflow of $j$, and the inflow of $i$ is not larger than the inflow of $j$. In this paper we characterize the ranking by outflow in which node $i$ is ranked 
at least as high as another node $j$ if and only if the outflow of $i$ is at least as high as the outflow of $j .{ }^{1}$ This ranking method generalizes the ranking by outdegree for directed graphs as characterized in van den Brink and Gilles [5]. We remind that a directed graph—or digraph—is a pair $(\mathrm{N}, \mathrm{D})$ where $\mathrm{D} \subseteq \mathrm{N} \times \mathrm{N}$ is a binary relation on the finite node set $\mathrm{N}$.

Applications of ranking methods are, for example, ranking teams in sports competitions, ranking alternatives in social choice theory (see, e.g., Sen [9]) and ranking firms in an industry. In Section 2 we introduce our axiomatization of the ranking by outflow method. Section 3 compares this method with the net flow method.

\section{Ranking by outflow}

The method of ranking by outflow is the ranking method $\mathrm{R}^{\text {out }}: \mathcal{W} \rightarrow \mathcal{P}$ which assigns to every $\omega \in \mathcal{W}$ a complete preorder $R^{\text {out }}(\omega) \in \mathcal{P}$ given by

$$
(i, j) \in R^{\text {out }}(\omega) \quad \text { if and only if } \quad \sigma_{i}^{\text {out }}(\omega) \geqq \sigma_{j}^{\text {out }}(\omega) .
$$

\subsection{An axiomatization}

For a ranking method represented by $\left\{\succcurlyeq_{\omega} \mid \omega \in \mathcal{W}\right\} \subseteq \mathcal{P}$ we introduce the following axiomatic properties. The first axiom is well-known from Bouyssou [2] and is a straightforward generalization of the corresponding axiom for digraphs given in Rubinstein [8]. For $\omega \in \mathcal{W}$ and a permutation $\pi: N \rightarrow N$ the permuted digraph $\pi \omega \in \mathcal{W}$ is given by $\pi \omega(\pi(i), \pi(j))=\omega(i, j)$ for all $(i, j) \in N \times N$.

Axiom 1 (Anonymity) For every $\omega \in \mathcal{W}$ and permutation $\pi: \mathrm{N} \rightarrow \mathrm{N}$ it holds that $i \succcurlyeq_{\omega} j$ if and only if $\pi(i) \succcurlyeq_{\pi \omega} \pi(j)$.

The second axiom is a straightforward generalization of Rubinstein's [8] corresponding axiom for digraphs and is implied by the stronger axiom of strong monotonicity used in Bouyssou [2]. It states that in pairwise comparison a node does better if it dominates more nodes.

Axiom 2 (Positive responsiveness) Let $\omega \in \mathcal{W}$ and let $\omega^{\prime} \in \mathcal{W}$ be such that for some pair $\mathrm{i}, \mathrm{h} \in \mathrm{N}$ there is a positive constant $\mathrm{c}>0$ such that:

$$
\omega^{\prime}(p, q)=\left\{\begin{array}{cl}
\omega(p, q)+c & \text { if }(p, q)=(i, h) \\
\omega(p, q) & \text { otherwise. }
\end{array}\right.
$$

\footnotetext{
${ }^{1}$ We refer to van den Brink and Gilles [4] for an axiomatization of the outflow as a relational power measure for weighted digraphs, also known as generalized score measure.
} 
If $i \succcurlyeq_{\omega} j$ then $i \succ_{\omega^{j} j}$.

The third axiom generalizes the independence axioms of van den Brink and Gilles [5] for digraphs and Rubinstein [8] for tournaments. It states that in pairwise comparison between nodes $i$ and node $j$, node $i$ is not doing worse in case its outflow does not decrease and j's outflow does not increase.

Axiom 3 (Outflow monotonicity) Let $\omega, \omega^{\prime} \in \mathcal{W}$ and $i, j \in N, i \neq j$, be such that $\omega^{\prime}(i, h) \geqq \omega(i, h)$ and $\omega^{\prime}(j, h) \leqq \omega(j, h)$ for all $h \in N$. If $i \succcurlyeq \omega j$ then $i \succcurlyeq \omega^{\prime} j$.

Note that outflow monotonicity implies that the order between two nodes does not change as long as their outflows do not change, i.e. if $\omega, \omega^{\prime} \in \mathcal{W}$ and $i, j \in N, i \neq j$, are such that $\omega^{\prime}(i, h)=\omega(i, h)$ and $\omega^{\prime}(j, h)=\omega(j, h)$ for all $h \in N$, then $i \succcurlyeq_{\omega} j$ if and only if $i \succcurlyeq_{\omega^{\prime}} j$.

Van den Brink and Gilles [5] show that these three axioms applied to digraphs characterize the ranking by outdegree for digraphs. However, these axioms do not characterize the ranking by outflow for weighted digraphs. We give an example to illustrate this in Section 2.2. Therefore, we need one more axiom which states that if we add two weighted digraphs and the pairwise ranking between two nodes is the same in both weighted digraphs, then this ranking is also the same in the 'sum' weighted digraph. For $\omega, \omega^{\prime} \in \mathcal{W}$, we define $\left(\omega+\omega^{\prime}\right) \in \mathcal{W}$ by $\left(\omega+\omega^{\prime}\right)(i, j):=$ $\omega(i, j)+\omega^{\prime}(i, j)$ for all $(i, j) \in N \times N$.

Axiom 4 (Order preservation) Let $\omega, \omega^{\prime} \in \mathcal{W}$ and let $i, j \in N$. If $i \succcurlyeq_{\omega} j$ and $i \succcurlyeq_{\omega^{\prime}} j$ then $i \succcurlyeq_{\left(\omega+\omega^{\prime}\right)} j$.

Next we state our main result.

Theorem A ranking method on $\mathcal{W}$ is equal to the method of ranking by outflow if and only if it satisfies anonymity, positive responsiveness, outflow monotonicity and order preservation.

Proof. We leave it to the reader to check that the method of ranking by outflow satisfies the four stated axioms.

To show the reverse, let $\left\{\succcurlyeq_{\omega} \mid \omega \in \mathcal{W}\right\} \subseteq \mathcal{P}$ represent a ranking method that satisfies the four stated axioms. Let $\omega \in \mathcal{W}$ and $i, j \in \mathrm{N}$ be arbitrary. For every $\omega \in \mathcal{W}$ we introduce the following

$$
\mathrm{m}(\omega):=\min \{\omega(h, g) \mid(h, g) \in \mathrm{N} \times \mathrm{N} \text { and } \omega(h, g)>0\}
$$




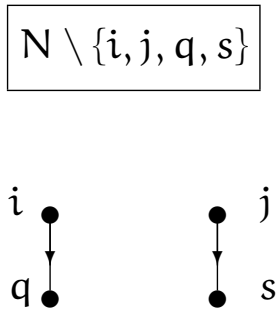

$\mathrm{q} \neq \mathrm{s}$

$$
N \backslash\{i, j, q\}
$$

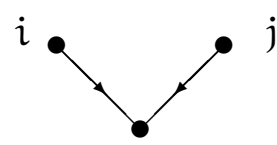

$q=s$

Figure 1: Weighted digraph $\omega^{k}$ in case $(i, j) \notin\{(p, q),(r, s)\}$.

$$
M(\omega):=\{(h, g) \in N \times N \mid \omega(h, g)=m(\omega)\}
$$

We first prove that $i \succcurlyeq_{\omega} j$ if $\sigma_{i}^{\text {out }}(\omega) \geqq \sigma_{j}^{\text {out }}(\omega)$. We distinguish the following two cases.

Case A. We first consider that $\sigma_{i}^{\text {out }}(\omega)=\sigma_{j}^{\text {out }}(\omega)$.

To show that $i \sim_{\omega} j$, we consider the weighted digraph $\widehat{\omega} \in \mathcal{W}$ given by

$$
\widehat{\omega}(h, g)=\left\{\begin{array}{cl}
\omega(h, g) & \text { if } h \in\{i, j\}, g \in N \\
0 & \text { otherwise. }
\end{array}\right.
$$

We may suppose without loss of generality that $\omega(i, j) \geqq \omega(j, i)$.

Next we construct a collection of weighted digraphs $\left\{\omega^{k}\right\}_{0 \leqq k \leqq t}$ for some finite $t \in \mathbb{N}$ such that nodes $i$ and $j$ are 'similar' in each of these digraphs, and thus we can apply anonymity to conclude that $i \sim_{\omega^{k}} j$ in all those digraphs. We construct this collection of weighted digraphs using the following procedure.

STEP 1 Let $\omega^{0}$ be given by

$$
\omega^{0}(h, g)=\left\{\begin{array}{cl}
\omega(j, i) & \text { if }(h, g) \in\{(i, j),(j, i)\} \\
0 & \text { otherwise }
\end{array}\right.
$$

Anonymity of $\succcurlyeq$ clearly implies that $i \sim \omega^{0} j$. Now let $\bar{\omega}^{1} \in \mathcal{W}$ be given by

$$
\bar{\omega}^{1}(h, g):=\left\{\begin{array}{cl}
\widehat{\omega}(h, g)-\omega(j, i) & \text { if }(h, g) \in\{(i, j),(j, i)\} \\
\widehat{\omega}(h, g) & \text { otherwise }
\end{array}\right.
$$

Note that $\bar{\omega}^{1}(j, i)=0$ and $\bar{\omega}^{1}(i, j) \geqq 0$.

Let $k=0$. 
STEP $2 \operatorname{IF}\left\{(h, g) \in N \times N \mid \bar{\omega}^{k+1}(h, g)>0\right\}=\emptyset$ THEN let $t=k$ and STOP.

ELSE since $\sigma_{i}^{\text {out }}(\widehat{\omega})=\sigma_{j}^{\text {out }}(\widehat{\omega})$ and $\sigma_{i}^{\text {out }}\left(\omega^{\mathrm{m}}\right)=\sigma_{j}^{\text {out }}\left(\omega^{\mathrm{m}}\right)$ for all $0 \leqq m \leqq k$, we know that there must exist at least one $h \in N \backslash\{i\} \operatorname{such}$ that $\bar{\omega}^{k+1}(i, h)>0$ and at least one $g \in N \backslash\{i, j\}$ such that $\bar{\omega}^{k+1}(j, g)>0$.

Let $k=k+1$ and GO TO STEP 3 .

STEP 3 Take a $(p, q) \in M\left(\bar{\omega}^{k}\right)$. (Note that $p \in\{i, j\}$.) Since $\sigma_{i}^{\text {out }}(\widehat{\omega})=\sigma_{j}^{\text {out }}(\widehat{\omega})$ and $\sigma_{i}^{\text {out }}\left(\omega^{m}\right)=\sigma_{j}^{\text {out }}\left(\omega^{m}\right)$ for all $0 \leqq m<k$ there exists an $(r, s) \in N \times N$ such that $\{r\}=\{i, j\} \backslash\{p\}$ and $\omega(r, s)>0$.

Let $\bar{\omega}^{k+1} \in \mathcal{W}$ be given by

$$
\bar{\omega}^{k+1}(h, g)=\left\{\begin{array}{cl}
\bar{\omega}^{k}(h, g)-m\left(\bar{\omega}^{k}\right) & \text { if }(h, g) \in\{(p, q),(r, s)\} \\
\bar{\omega}^{k}(h, g) & \text { otherwise. }
\end{array}\right.
$$

Clearly $\bar{\omega}^{k+1}(p, q)=0$ and $\bar{\omega}^{k+1}(h, g) \geqq 0$ for all $(h, g) \neq(p, q)$, thus $\omega^{k+1} \in$ $\mathcal{W}$.

Next we distinguish between the following two cases:

IF $(i, j) \notin\{(p, q),(r, s)\}$ THEN let $\omega^{k}$ be given by

$$
\omega^{k}(h, g):=\left\{\begin{array}{cl}
m\left(\bar{\omega}^{k}\right) & \text { if }(h, g) \in\{(p, q),(r, s)\} \\
0 & \text { otherwise. }
\end{array}\right.
$$

The weighted digraph $\omega^{k}$ is illustrated in Figure 1 with $p=i$ and $r=j$, in case $\mathrm{q} \neq \mathrm{s}$ respectively if $\mathrm{q}=\mathrm{s}$. Note that anonymity implies that $i \sim \omega^{k} j$.

GO TO STEP 2.

$\operatorname{ELSE}(i, j) \in\{(p, q),(r, s)\}$. Suppose that $(i, j)=(p, q)$ and thus $r=j$. Then let $\omega^{k}$ be given by

$$
\omega^{k}(h, g):=\left\{\begin{array}{cl}
m\left(\bar{\omega}^{k}\right) & \text { if }(h, g) \in\{(i, j),(j, s),(s, i)\} \\
0 & \text { otherwise. }
\end{array}\right.
$$

The weighted digraph $\omega^{k}$ is illustrated in Figure 2. Anonymity also implies that $i \sim{ }_{\omega^{k}} j$ in this case.

If $(i, j)=(r, s)$ then we do the same but with $s$ replaced by q. Go TO STEP 2.

Since $\sigma_{i}^{\text {out }}(\widehat{\omega})=\sigma_{j}^{\text {out }}(\widehat{\omega})$ this procedure leads to a collection of weighted digraphs $\left\{\omega^{k}\right\}_{0 \leqq k \leqq t}$ which have been constructed such that nodes $i$ and $j$ are 'similar' in each 

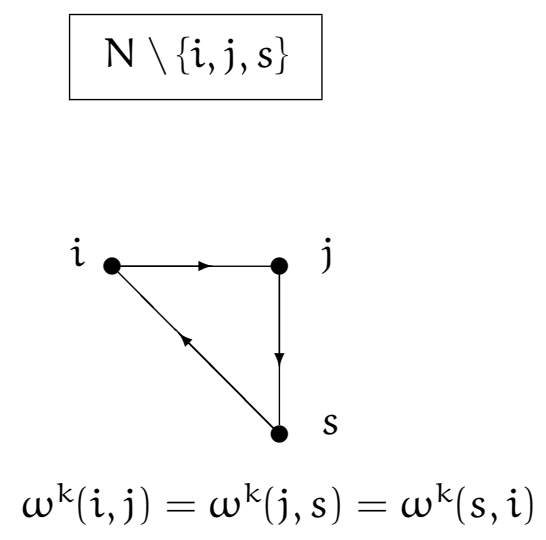

Figure 2: Weighted digraph $\omega^{k}$ in case $(i, j) \in\{(p, q),(r, s)\}$.

of these weighted digraphs.

Anonymity then implies that for every $\omega^{k}, 0 \leqq k \leqq t$, it holds that $i \sim \omega^{k} j$.

Now let $\omega^{\prime} \in \mathcal{W}$ be given by $\omega^{\prime}(i, j):=\sum_{k=0}^{t} \omega^{k}(i, j)$ for all $(i, j) \in N \times N$.

Order preservation then implies that $i \sim \omega_{\omega^{\prime}} j$.

If $\omega(i, j)=\omega(j, i)$ then $\omega^{0}(i, j)=\omega^{0}(j, i)=\omega(i, j)$ and $\bar{\omega}^{k}(i, j)=\bar{\omega}^{k}(j, i)=0$ for all $1 \leqq k \leqq t$ and thus case 2 in Step 3 cannot occur. But then $\omega^{\prime}=\widehat{\omega}$ and thus $i \sim \widehat{\omega} j$.

Else $\omega(i, j)>\omega(j, i)$, and then there is some $s \in N \backslash\{i\}$ and some positive constant c $>0$ such that ${ }^{2}$

$$
\omega^{\prime}(h, g)=\left\{\begin{array}{cl}
\widehat{w}(h, g)+c & \text { if }(h, g)=(s, i) \\
\widehat{\omega}(h, g) & \text { otherwise. }
\end{array}\right.
$$

Outflow monotonicity together with $i \sim_{\omega^{\prime}} j$ then yields that in this case also $i \sim \widehat{\omega} j$. Thus, $i \sim \widehat{\omega} j$, and since $\omega(i, g)=\widehat{\omega}(i, g)$ and $\omega(j, g)=\widehat{\omega}(j, g)$ for all $g \in N$, outflow monotonicity implies that $i \sim_{\omega} j$. Thus, we conclude that $i \sim_{\omega} j$ if $\sigma_{i}^{\text {out }}(\omega)=$ $\sigma_{j}^{\text {out }}(\omega)$.

Case B. Next we turn to the case that $\sigma_{i}^{\text {out }}(\omega) \neq \sigma_{j}^{\text {out }}(\omega)$.

Without loss of generality we may assume that $\sigma_{i}^{\text {out }}(\omega)>\sigma_{j}^{\text {out }}(\omega)$. Then there exists a weighted digraph $\widetilde{\omega} \in \mathcal{W}$ satisfying the following conditions:

- $\widetilde{\omega}(h, g) \leqq \omega(h, g)$ for all $(h, g) \in N \times N$;

- $\widetilde{\omega}(h, g)=\omega(h, g)$ for all $h \in N \backslash\{i\}$ and $g \in N$;

- $\sigma_{i}^{\text {out }}(\widetilde{\omega})=\sigma_{j}^{\text {out }}(\widetilde{\omega})$.

\footnotetext{
${ }^{2}$ We refer to an illustration of this case in Figure 2.
} 
As shown above it follows from anonymity, outflow monotonicity and order preservation that $i \sim \widetilde{\omega} j$. Repeated application of positive responsiveness then yields that $i \succ_{\omega} j$.

From Cases A and B it follows that $i \succcurlyeq_{\omega} j$ if $\sigma_{i}^{\text {out }}(\omega) \geqq \sigma_{j}^{\text {out }}(\omega)$.

To show the reverse, note that Case B also implies that $j \succ_{\omega} i$ if $\sigma_{i}^{\text {out }}(\omega)<\sigma_{j}^{\text {out }}(\omega)$. With Case A this yields $\sigma_{i}^{\text {out }}(\omega) \geqq \sigma_{j}^{\text {out }}(\omega)$ if $i \succcurlyeq_{\omega} j$.

Thus, if a ranking method satisfies the four axioms then it has to be the ranking by outflow.

\subsection{Logical independence of the axioms}

The logical independence of the four axioms discussed above follows from the following four alternative ranking methods. For each method we show which axiom it does not satisfy using the following example. We leave it for the reader to verify the necessity of the other axioms.

Consider the weighted digraph $\omega_{0}$ on $N=\{1, \ldots, 4\}$ given by:

$$
\omega_{0}(i, j)= \begin{cases}1 & \text { if }(i, j) \in\{(1,3),(1,4),(2,3),(2,4)\} \\ 2 & \text { if }(i, j)=(3,4) \\ 0 & \text { otherwise }\end{cases}
$$

Then $\sigma^{\text {out }}\left(\omega_{0}\right)=(2,2,2,0)$ and the ranking by outflow yields: $1 \sim_{\omega_{0}} 2 \sim_{\omega_{0}} 3 \succ_{\omega_{0}} 4$.

1. Consider the ranking method $R^{1}$ given by

$$
i \succ_{\omega}^{1} j \text { if and only if }\left\{\begin{array}{l}
\text { either }\left[\sigma_{i}^{\text {out }}(\omega)>\sigma_{j}^{\text {out }}(\omega)\right] \\
\text { or }\left[\sigma_{i}^{\text {out }}(\omega)=\sigma_{j}^{\text {out }}(\omega) \text { and } i<j\right]
\end{array}\right.
$$

This ranking method satisfies all four axioms except anonymity. For the weighted digraph $\omega_{0}$ given above it yields that $1 \succ_{\omega_{0}}^{1} 2$ although $\omega_{0}(1, h)=\omega_{0}(2, h)$ and $\omega_{0}(h, 1)=\omega_{0}(h, 2)$ for all $h \in N$.

2. Consider the ranking method $R^{2}$ given by: $i \sim_{\omega}^{2} j$ for all $i, j \in N$. This ranking method satisfies all four axioms except positive responsiveness. Consider the weighted digraph $\omega_{0}$ given above and the weighted digraph $\omega^{\prime}$ given by

$$
\omega^{\prime}(i, j)=\left\{\begin{array}{cl}
2 & \text { if }(i, j)=(1,3) \\
\omega_{0}(i, j) & \text { otherwise }
\end{array}\right.
$$

If positive responsiveness is satisfied then it must hold that $1 \succ_{\omega^{\prime}}^{2} 2$ since $1 \sim_{\omega}^{2} 2$. But $1 \sim_{\omega^{\prime}}^{2} 2$. 
3. We define the inflow of node $i$ in $\omega \in \mathcal{W}$ as $\sigma_{i}^{i n}(\omega)=\sum_{j \in N} \omega(j, i)$. Next, the net flow of node $i$ in weighted digraph $\omega \in \mathcal{W}$ is given by

$$
\sigma_{i}^{\text {net }}(\omega)=\sigma_{i}^{\text {out }}(\omega)-\sigma_{i}^{\text {in }}(\omega)
$$

Consider the ranking method $\mathrm{R}^{3}$ given by

$$
i \succcurlyeq_{\omega}^{3} j \text { if and only if } \sigma_{i}^{\text {net }}(\omega) \geqq \sigma_{i}^{\text {net }}(\omega) \text {. }
$$

This ranking method satisfies all four axioms except outflow monotonicity. Consider $\omega_{0}$ given above and $\omega^{\prime}$ given by

$$
\omega^{\prime}(i, j)=\left\{\begin{array}{cl}
1 & \text { if }(i, j)=\{(3,2)\} \\
\omega_{0}(i, j) & \text { otherwise }
\end{array}\right.
$$

If $\mathrm{R}^{3}$ satisfies outflow monotonicity, then it must hold that $1 \sim_{\omega^{\prime}}^{3} 2$ since $1 \sim_{\omega_{0}}^{3} 2$ and $\omega^{\prime}$ is as described in Axiom 3. But $1 \succ_{\omega^{\prime}}^{3} 2$.

4. Let $\bar{\sigma}: \mathcal{W} \rightarrow \mathbb{R}^{N}$ be given by:

$$
\bar{\sigma}_{i}(\omega):=\#\{j \in N \mid \omega(i, j)>0\} \text { for all } i \in N \text { and } \omega \in \mathcal{W} \text {. }
$$

Thus $\bar{\sigma}$ assigns to every weighted digraph $\omega$ the outdegree of the underlying (non-weighted) digraph $\mathrm{D}$ where $\mathrm{D}=\{(i, j) \in \mathrm{N} \times \mathrm{N} \mid \boldsymbol{\omega}(i, j)>0\}$. Now, consider the ranking method $\mathrm{R}^{4}$ given by

$$
i \succcurlyeq_{\omega}^{4} j \text { if and only if } \bar{\sigma}_{i}(\omega) \cdot \sigma_{i}^{\text {out }}(\omega) \geqq \bar{\sigma}_{j}(\omega) \cdot \sigma_{j}^{\text {out }}(\omega) \text {. }
$$

This ranking method satisfies all four axioms except order preservation. Consider the weighted digraphs $\omega^{1}$ and $\omega^{2}$ given by

$$
\omega^{1}(i, j)= \begin{cases}1 & \text { for all }(i, j) \in\{(1,3),(2,3),(2,4),(3,4)\} \\ 0 & \text { otherwise }\end{cases}
$$

and

$$
\omega^{2}(i, j)= \begin{cases}1 & \text { for all }(i, j) \in\{(1,4),(3,4)\} \\ 0 & \text { otherwise }\end{cases}
$$

Then $3 \succcurlyeq_{\omega^{1}}^{4} 1$ and $3 \succcurlyeq_{\omega^{2}}^{4} 1$. Since $\left(\omega^{1}+\omega^{2}\right)=\omega_{0}$, if order preservation is satisfied it must hold that $3 \succcurlyeq_{\omega_{0}}^{4} 1$. But $1 \succ_{\omega_{0}}^{4} 3$. 


\section{A comparison with the net flow method}

The method of ranking by outflow generalizes the method of ranking by outdegree for digraphs which is axiomatized in van den Brink and Gilles [5]. The ranking method $R^{3}$ given at the end of the previous section is also known as the method of ranking by net flow and is characterized in Bouyssou [2]. Applied to directed graphs, this method boils down to the ranking by Copeland score. Both ranking by outflow and ranking by net flow methods generalize the ranking by Copeland score for tournaments as characterized in Rubinstein [8], see van den Brink and Gilles [5].

We first show that the ranking by outflow and net flow are different on $\mathcal{W}$. We illustrate this with an application considering social welfare ranking methods. Preferences of an individual a over a set of $n$ alternatives $N=\{1, \ldots, n\}$ can be represented by a binary relation $\succeq_{a}$ on $N$. We denote $i \succeq_{a} j$ if individual a weakly prefers alternative $i$ to alternative $j$. For a society of individuals represented by a set of individuals $I=\{1, \ldots, m\}$, a preference profile $p$ is an $m$-tuple of such preference relations. A social welfare ranking method assigns to every preference profile a complete preorder on the set of alternatives.

As a specific example, consider a set of four individuals $\{a, b, c, d\}$, a set of three alternatives $\{1,2,3\}$ and the preference profile $\left\{1 \succ_{\mathrm{a}} 2 \succ_{\mathrm{a}} 3,1 \succ_{\mathrm{b}} 2 \succ_{\mathrm{b}} 3,3 \succ_{\mathrm{c}}\right.$ $\left.1 \succ_{\mathrm{c}} 2,2 \sim_{\mathrm{d}} 3 \succ_{\mathrm{d}} 1\right\}$.

The corresponding weighted majority digraph is given by $\omega_{\mathrm{p}}: \mathrm{N} \times \mathrm{N} \rightarrow \mathbb{R}$ with

$$
\omega_{p}(i, j)=\#\left\{a \in I \mid i \succeq_{a} j\right\}-\#\left\{a \in I \mid j \succeq_{a} i\right\} .
$$

For the specified example, $\omega_{p}(1,2)=2, \omega_{p}(2,3)=1$, and $\omega_{p}(i, j)=0$ otherwise. The outflows, respectively net flows, are given by $\sigma^{\text {out }}\left(\omega_{p}\right)=(2,1,0)$, respectively $\sigma^{\text {net }}\left(\omega_{p}\right)=(2,-1,-1)$. So, according to the ranking method by outflow node 2 is ranked higher than node 3 , while according to the ranking by net flow nodes 2 and 3 are ranked equally.

Bouyssou [2] characterized the method of ranking by net flow using anonymity, strong monotonicity and independence of 2- and 3-cycles. ${ }^{3}$ Strong monotonicity consists of positive responsiveness and the reverse negative responsiveness, which requires that if $i$ is ranked at least as high as $j$, then increasing the inflow of $j$ makes $i$ being ranked higher than $j$. Independence of 2- and 3-cycles means that deleting or

\footnotetext{
${ }^{3}$ Henriet [7] characterizes the net flow ranking method restricted to the class of so-called "complete 2-digraphs". A complete 2-digraph is a weighed digraph with weights 0,1 and 2 on the arcs such that the sum of the weight of an arc and the weight of the reverse arc is exactly 2 .
} 
adding a cycle of length 2 or 3 to a weighted digraph does not change the ranking of the nodes. The ranking by net flow does not satisfy outflow monotonicity as defined in Section 2. On the other hand, the ranking by outflow does not satisfy independence of 2- or 3-cycles nor negative responsiveness.

We extend our argument made in van den Brink and Gilles [5] that the axioms that are presented in this paper are more in line with Rubinstein [8]'s axioms on tournaments. A tournament is a weighted digraph $\omega$ such that $\omega(i, j) \in\{0,1\}$ and $\omega(i, j)=1$ if and only if $\omega(j, i)=0$. First, we emphasize that independence of 2- and 3-cycles is not well defined on the class of complete digraphs. Namely, if such a 2- or 3-cycle is removed from a complete digraph, the resulting digraph might no longer be complete. This prevents a thorough comparison of Bouyssou's axiomatization of the ranking by net flow with Rubinstein's axiomatization of the ranking by Copeland score on the class of tournaments.

A second argument in favor of the ranking by outflow is the fact that outflow monotonicity generalizes Rubinstein's independence axiom, while independence of 2 - or 3-cycles does not ${ }^{4}$. To show this we formally generalize independence of 2 or 3-cycles and Rubinstein's independence of irrelevant arcs for weighted digraphs.

Axiom 5 (Independence of 2- or 3-cycles) Let $\omega, \omega^{\prime} \in \mathcal{W}$ be such that $\omega^{\prime}(h, g)-$ $\omega(h, g)=\omega^{\prime}(g, h)-\omega(g, h)$ for some $h, g \in N$, or $\omega^{\prime}(h, g)-\omega(h, g)=\omega^{\prime}(g, f)-$ $\omega(g, f)=\omega^{\prime}(f, h)-\omega(f, h)$ for some $h, g, f \in N$. Then $i \succcurlyeq_{\omega} j$ if and only if $i \succcurlyeq_{\omega^{\prime}} j$ for all $i, j \in N$.

Axiom 6 (Independence of irrelevant arcs) Let $\omega, \omega^{\prime} \in \mathcal{W}$ and $i, j \in N$ be such that $\omega(i, h)=\omega^{\prime}(i, h), \omega(h, i)=\omega^{\prime}(h, i), \omega(j, h)=\omega^{\prime}(j, h)$, and $\omega(h, j)=\omega^{\prime}(h, j)$ for all $h \in N$. Then $i \succcurlyeq_{\omega} j$ if and only if $i \succcurlyeq_{\omega^{\prime}} j$.

Next we compare independence of irrelevant arcs with outflow monotonicity and independence of 2- or 3-cycles.

Proposition Let $\mathrm{R}$ be a ranking method on $\mathcal{W}$.

(a) If $\mathrm{R}$ satisfies outflow monotonicity then $\mathrm{R}$ satisfies independence of irrelevant arcs.

(b) The axioms of independence of 2- or 3-cycles and independence of irrelevant arcs do not imply one another.

\footnotetext{
${ }^{4}$ In van den Brink and Gilles [5] the same is shown for the ranking by outdegree for digraphs.
} 
Proof. As usual we represent the ranking method $\mathrm{R}$ by $\left\{\succcurlyeq_{\omega} \mid \omega \in \mathcal{W}\right\} \subseteq \mathcal{P}$. Assertion (a) follows trivially from the definitions of the independence axioms under consideration.

We proceed to show assertion (b). The ranking by outflow satisfies independence of irrelevant arcs but does not satisfy independence of 2- or 3-cycles.

Next, consider the ranking method $R^{5}$ given by

$$
i \succcurlyeq_{\omega}^{5} j \text { if and only if }\left\{\begin{array}{l}
\text { either }\left[\sigma_{i}^{\text {net }}(\omega)=\max _{h \in N} \sigma_{h}^{\text {net }}(\omega)\right] \\
\operatorname{or}\left[\max \left\{\sigma_{i}^{\text {net }}(\omega), \sigma_{j}^{\text {net }}(\omega)\right\}<\max _{h \in N} \sigma_{h}^{\text {net }}(\omega)\right]
\end{array}\right.
$$

This ranking partitions the set of nodes in two subsets. The "winners" are the ones with the highest net flow. This ranking method satisfies independence of 2- or 3-cycles, but does not satisfy independence of irrelevant arcs.

\section{References}

[1] N. Bourbaki, Elements of Mathematics: Theory of Sets (Hermann Publishers, Paris, 1968).

[2] D. Bouyssou , Ranking Methods Based on Valued Preference Relations: A Characterization of the Net Flow Method, European Journal of Operational Research 60 (1992) 61-67.

[3] D. Bouyssou and P. Perny, Ranking Methods for Valued Preference Relations, European Journal of Operational Research 61 (1992) 186-194.

[4] R. van den Brink and R.P. Gilles, Measuring Domination in Directed Networks, Social Networks, 22 (2000) 141-157.

[5] R. van den Brink and R.P. Gilles, Ranking by Outdegree for Directed Graphs, Discrete Mathematics, 271 (2002) 261-270.

[6] A.H. Copeland, A 'Reasonable' Social Welfare Function, Mimeographed notes, University of Michigan Seminar on Applications of Mathematics to the Social Sciences, 1951.

[7] D. Henriet, The Copeland Choice Function: An Axiomatic Characterization, Social Choice and Welfare 2 (1985) 49-63.

[8] A.Rubinstein, Ranking the Participants in a Tournament, SIAM Journal of Applied Mathematics 38 (1980) 108-111.

[9] A.K. Sen, Collective Choice and Social Welfare (North Holland Publishing Company, Amsterdam, The Netherlands, 1979). 